\title{
INNOVACIÓN, DEMOCRATIZACIÓN Y MEJORA DE LA DOCENCIA UNIVERSITARIA EN EL MARCO DE LA SOCIEDAD DE LA INFORMACIÓN
}

\author{
Ramón Flecha y Josep $M^{a}$ Rotger \\ Universitat de Barcelona
}

\begin{abstract}
RESUMEN: La actual sociedad de la información ha supuesto enormes avances respecto al anterior modelo de la sociedad industrial. Sin embargo, la universidad sigue funcionando del mismo modo que lo hacía antes. Las capacidades de seleccionar la información más relevante de entre un enorme cúmulo de datos y de procesarla para aplicarla adecuadamente en cada situación resultan hoy mucho más necesarias que la posibilidad de almacenar conocimientos. En este contexto, es importante resaltar aquellas iniciativas que, a la luz de estas nuevas exigencias, se están llevando a la práctica para mejorar la docencia universitaria. Es el caso de aquellas asignaturas que promueven el uso de las bases de datos en la red o los intercambios a través de forums. El aumento de las interacciones entre alumnos, alumnas, profesorado e incluso otras personas interesadas en un campo concreto pueden enriquecer enormemente los proceso de enseñanza-aprendizaje, muy en la línea del aprendizaje dialógico. Apoyadas en las posibilidades que ofrecen las tecnologías de la información y la comunicación, estas propuestas contribuyen además a la democratización y transparencia de la institución universitaria, ya que establecen un marco más horizontal al cual todo el mundo puede acceder y participar en pie de igualdad.
\end{abstract}

\footnotetext{
ABSTRACT: The current information society have brought together many changes with regard to the former industrial society. However, the university continues functioning in the same way that it formerly used to. The capacities of selecting the most relevant information among a lot of data and processing it properly in every situation are today much more necessary than the possibility of storing knowledge. In this context, it is important to highlight those initiatives that are being implemented in order to improve university teaching according to these new requirements. It is the case of that subjects that promote the use of Internet database or exchanges through forums. The increasing of interactions among pupils, teachers or even other people interested in a certain field can enrich enormously the teaching-learning process, very much in line with the notion of dialogic learning. Supported on the possibilities offered by the information and communication technologies, these proposals also
} 
contribute to the democratization and transparency of the university institution, since they establish a more horizontal framework that everybody can accede and participate in on an equal foot.

PALABRAS CLAVE: Sociedad de la información, universidad, innovación, docencia, democratización, diálogo.

KEYWORDS: Information society, university, innovation, teaching, democratization, dialogic learning.

\section{Introducción}

Si pudiéramos observar por una ventana el aspecto de nuestras ciudades hace treinta años, o qué pasaba en los lugares de trabajo entonces, y por otra ventana observáramos la misma escena hoy, veríamos diferencias notables en la organización, en las relaciones personales, en los recursos, etc. El agotamiento del modelo de sociedad industrial, junto con el enorme desarrollo de las tecnologías de la información y la comunicación (en adelante TIC), promovieron un cambio de paradigma social en los años setenta que dio paso a la sociedad de la información. Sin embargo, si miráramos también por la ventana de un aula universitaria hace treinta años, veríamos una multitud de alumnos y alumnas tomando apuntes y un profesor o profesora explicando la lección en la pizarra y subida a una tarima. Paradójicamente, en muchas de nuestras universidades aún podemos contemplar hoy una escena similar. ¿Cómo es posible que la docencia continúe impermeable a los cambios que operan en todos los ámbitos de nuestra sociedad? ¿Por qué las universidades, en la supuesta vanguardia de la ciencia, mantienen la docencia en la retaguardia? ¿Por qué no se hace uso de todos los recursos que ofrece la sociedad de la información para mejorar la enseñanza y el aprendizaje?

En este artículo explicamos en primer lugar los rasgos más significativos de la sociedad de la información. En el segundo apartado, se analizan algunas de las prácticas que están contribuyendo a la innovación y mejora de la calidad docente y que guardan estrecha relación con los cambios sociales que se han producido en el contexto social actual. En el tercer punto, nos centramos en cómo los procesos de democratización que se desarrollan en nuestros días tienen también su reflejo en la universidad y, por extensión, también en la calidad docente.

\section{La sociedad de la información}

El modelo industrial de desarrollo se asentaba sobre la transformación de materia prima en objetos o bienes de consumo, de manera que los recursos materiales eran fundamentales. La educación consistía en aprender unos contenidos específicos o un oficio. En la actual sociedad informacional, en cambio, la clave está en los recursos humanos $y$, en concreto, en cómo las personas gestionamos la información. De hecho, autores como Gorz, McLuhan, Beck, Castells o Sen coinciden en identificar la selección y el procesamiento de la información como el principal rasgo característico de este modelo de sociedad. Así pues, la materia prima por excelencia es ahora la información misma. Trabajamos sobre ella para producir más o para hacer más rápidos y más eficaces los sistemas y las tecnologías para su procesamiento. La 
educación se centra ahora en saber acceder, seleccionar y procesar esta información $y$, en definitiva, saber aprender a lo largo de la vida.

A finales del siglo XX, los grupos dominantes priorizaron la rápida introducción de la revolución informacional tomando como base el sector de la sociedad con recursos para hacerlo. De este modo, los países y las personas sin los conocimientos y las tecnologías que requiere el desarrollo de la sociedad de la información se vieron excluidos. Se generó así un proceso de polarización o dualización social que caracterizó la primera fase de desarrollo de la sociedad de la información (Flecha, Gómez, Puigvert 2001). Fue entonces, a partir de la guerra del petróleo de 1973, detonante del cambio de paradigma social, cuando el paro y la exclusión comenzaron a aparecer como crecientes problemas sociales. Al mismo tiempo, las ocupaciones con alto contenido informacional adquirieron una enorme importancia en la nueva economía. Se configuró así lo que Gorz (1986) denominó sociedad de los dos tercios, estratificada en tres sectores. En el primero, se encuentran las personas incluidas, con titulaciones académicas y puestos de trabajo estables. En el segundo, las personas explotadas, poco cualificadas y con ocupaciones precarias. En el tercer sector se incluyen las personas en situaciones laborales inestables, de paro o en prácticas de economía delictiva (Aubert et al., 1994).

Actualmente esta tendencia hacia la polarización social, en la que la sociedad se fragmenta en función del dominio de las nuevas competencias informacionales, se está viendo frenada por una dinámica diametralmente opuesta que apunta hacia una segunda fase de la sociedad de la información abierta a todos y todas. Por un lado, las grandes empresas del sector informacional pretenden extenderse por todo el mundo. El mejor ejemplo lo encontramos en Microsoft y su creador, Bill Gates, cuyos productos están llegando cada vez a más rincones del planeta. Por otro lado, los problemas creados por la desigualdad y la exclusión, junto con las presiones de los países excluidos y los movimientos sociales igualitarios, han conseguido despertar una cierta conciencia y defensa ciudadana de la sociedad de la información para todas las personas.

Cada vez son más las personas que quieren estudiar y que están aprendiendo a usar las tecnologías. Al mismo tiempo, muchas personas y movimientos sociales se están apropiando de las nuevas tecnologías para poder participar activamente en el desarrollo y en los beneficios de esta nueva sociedad. Los movimientos sociales han encontrado en las TIC la mejor herramienta para extender su voz. Por ejemplo, organizaciones como RAWA (Asociación Revolucionaria de las Mujeres de Afganistán) o el Forum Social Mundial utilizan los recursos que les ofrecen las nuevas tecnologías para difundir su mensaje, su clamor contra la opresión o sus propuestas transformadoras. Sus páginas web (http://www.portoalegre2003.org y http://www.rawa.org) superan los obstáculos institucionales y les permiten extender sus reivindicaciones a cualquier lugar, establecer foros para el intercambio de ideas y generar un primer nexo de unión con todos aquellos o aquellas que decidan apoyarles. Los gobiernos, además, se están mostrando muy permeables a las propuestas en la línea de la sociedad de la información para todas las personas. En este sentido, cabe citar el plan de acción e-Learning de la Comisión Europea, cuya primera finalidad es acelerar el despliegue en la Unión Europea de una infraestructura de calidad a costes accesibles (Comisión de las Comunidades Europeas 2001). 
La universidad no puede quedarse atrás en estos cambios. Si la educación superior debe ser un espacio de aprendizaje, acceso a información, intercambio de ideas y elaboración de propuestas innovadoras, las TIC ofrecen enormes posibilidades para reavivar estas funciones. La relativa facilidad con la que todos y todas podemos hoy acceder a la red y al uso de tecnologías multimedia debe suponer un impulso significativo para que más personas puedan acceder a la formación universitaria y para que ésta gane en dinamismo, frescura, transparencia y democracia, tanto en su funcionamiento interno como en la realidad del aula. De hecho, algunas de las prácticas que se están Ilevando a cabo se sitúan en esta línea y están contribuyendo sensiblemente a la mejora de la calidad docente en la universidad.

\section{Innovación y mejora de la calidad docente}

En la actual sociedad de la información, el aprendizaje no depende tanto de lo que ocurre en el aula como de la correlación entre lo que ocurre en el aula, el domicilio, la calle, la influencia de los medios de comunicación, etc. (Elboj et al. 2002: 75).

En la actual sociedad de la información los o las discentes no necesitan tanto un profesorado que les transmita conocimientos como alguien que les ayude a orientarse en este mar de informaciones provenientes de todos los ámbitos en el que se ven inmersos. Así, ellos y ellas ya tienen la posibilidad de acceder a mucha información. Como ya hemos apuntado, lo que hoy requiere la sociedad es saber acceder a esta información y saber escoger y gestionar la más relevante en cada momento para poder aplicar en una situación dada. Es lo que, de otro modo, se expresa en el Informe Delors cuando se define el significado de aprender a conocer:

Aprender para conocer supone, en primer término, aprender a aprender (...). El joven debe aprender a concentrar la atención en las cosas y en las personas. La vertiginosa sucesión de informaciones en los medios de comunicación (...) requiere una permanencia y una profundización de la información captada. (Delors 1996: 98)

A los jóvenes de nuestros días no se les exigirá en sus futuras profesiones que conozcan absolutamente todos los contenidos relacionados con su trabajo, sino que sepan encontrar toda esa información en el momento en que sea necesaria. A ningún profesional de la educación le van a pedir que no consulte ningún libro o que no realice búsquedas en Internet para elaborar una unidad de programación, desarrollar nuevas tareas o hacer un diagnóstico para un plan de actuación... Entonces, ¿por qué seguimos planteando las evaluaciones de manera que nuestro alumnado tenga que resolver casos sin tener acceso a ninguna fuente de información? ¿Por qué no proporcionar a través de nuestra docencia las estrategias de trabajo y las fuentes que tendrán que consultar como profesionales? Seguimos funcionando con la imagen que se veía en esa ventana hace treinta años, mientras el mundo va a otra velocidad.

A pesar de que, en general, la universidad no se ha mostrado sensible a esta nueva realidad social, sí que existen algunas iniciativas muy interesantes en la docencia universitaria que se enmarcan plenamente en la sociedad de la información. Es decir, que proponen unos contenidos, una metodología y utiliza unos recursos para la enseñanza que permiten al alumnado adquirir las habilidades requeridas en el modelo actual de sociedad. Las TIC desempeñan un papel esencial en estas prácticas innovadoras, puesto que enriquecen la calidad de la docencia mediante la aplicación de 
múltiples recursos como lo que, en algunas universidades Ilamamos "dossieres electrónicos". Se trata de un espacio virtual del que dispone el profesorado para poder "colgar" documentación relacionada con una asignatura, para crear links con otros espacios de la red, para informar e incluso tutorizar y realizar debates con el alumnado a través de un forum.

Otro uso de las TIC al servicio de la calidad docente lo constituyen las bases de datos en la red, que permiten acceder a informaciones ya seleccionadas por parte de las comunidades científicas. Tanto alumnado como profesorado ya no dependen únicamente de un libro o de un dossier de lecturas básicas; además pueden acceder a bases de datos como Sociological Abstracts (o Sociofile) y ver qué autores y teorías están más citadas en estos momentos y encontrar publicaciones con aportaciones clave para la asignatura y los trabajos de clase. Por ejemplo Sociofile recoge, en el ámbito de las ciencias sociales, las informaciones de más de 2000 revistas de 55 países y en 30 lenguas diferentes. Así mismo, en el ámbito de las ciencias de la educación, la base de datos ERIC ofrece un amplísimo abanico de artículos sobre educación y temas afines. La información que éstas y otras bases de datos ponen a disposición de todo el mundo contribuyen enormemente a la democratización y horizontalidad de la comunicación científica, ya que elimina los posibles sesgos que podía haber en el pasado al transmitirse la información en estricto orden jerárquico. Las bases de datos constituyen, además, un inmejorable instrumento para desarrollar las habilidades de selección y procesamiento de la información, puesto que el estudiantado debe nadar entre un inmenso mar de datos para hallar y reelaborar aquellos que resulten realmente relevantes en su trabajo. Esto, junto con la posibilidad de saber en pocos segundos quiénes son los autores más referenciados en un área concreta, hace que las bases de datos ayuden a dotar de calidad tanto a los resultados que presenta el alumnado como al propio proceso de enseñanzaaprendizaje.

La utilización de estos recursos puede contribuir en mayor o menor medida a la mejora de la docencia universitaria. Pero queremos destacar, entre todos ellos, el enorme potencial de los forums virtuales de debate para enriquecer los procesos de enseñanza y aprendizaje. Tanto si se trata de forums propios de una asignatura como de otros relacionados con el mismo campo de estudio, éstos ofrecen infinitas posibilidades de interacción para las personas que participan. A menudo, los debates iniciados en el aula continúan durante varios días en el forum, o las preguntas o reflexiones que nunca se hicieron en clase despiertan intereses y pasiones a través del ordenador. Cuantas más personas estén conectadas, más posibilidades de interacción y, por consiguiente, más posibilidades de enriquecerse con las aportaciones y puntos de vista de los demás. Más y nuevas interacciones implican más y nuevos aprendizajes.

Esta práctica entronca directamente con la noción de aprendizaje dialógico, que considera que las personas construimos nuestros propios significados (es decir, aprendemos) a través de las interacciones con los demás mediante el diálogo intersubjetivo. En cambio, se distancia de la noción de aprendizaje significativo, que entiende la construcción de significados como un proceso cognitivo individual, obviando su carácter social. Pero las interacciones o el diálogo no impulsan el aprendizaje en cualquier circunstancia: el diálogo debe ser igualitario, de manera que las personas expongan sus argumentos en condiciones de igualdad y con la voluntad de entenderse desde pretensiones de validez (Habermas, 1987). Es decir, que no se pretende imponer el propio punto de vista, sino interaccionar de forma democrática, 
horizontal y para llegar a un consenso. Aunque estas condiciones se pueden dar y de hecho se dan en muchos otros casos, los forums de las asignaturas suponen un marco inmejorable para aprender mediante la interacción con el resto de compañeros y compañeras, ya que el profesorado, suponiendo que tenga voluntad de hacerlo, siempre puede reorientar el diálogo en esta dirección.

La aplicación de un sistema de evaluación en base a créditos europeos puede beneficiarse de, e impulsar a la vez, estas y otras prácticas de trabajo semi-presencial. El reconocimiento del trabajo propio y los conocimientos adquiridos por el estudiantado, en relación con una asignatura concreta, en contextos distintos al aula, es en este sentido un avance. Así, desde el inicio hasta el final de la asignatura, podemos registrar tanto las horas de clase presencial como las invertidas en actividades relacionadas tales como la consulta bibliográfica, la búsqueda en Internet, las intervenciones en el forum de la asignatura, la lectura de libros relacionados con el contenido de la materia, la participación en debates teóricos presenciales, la recogida de información de revista, diarios o documentos, la participación en otros forums de debate, en jornadas, conferencias, congresos, etc.

\section{Democratización de la universidad y promoción}

La sociedad de la información es el marco en el que el diálogo se extiende a cada vez más ámbitos de la experiencia humana, y ciertas prácticas o realidades que antes eran incuestionables ahora son sometidas al debate y al consenso entre las personas. Es por esto que hablamos de giro dialógico de la sociedad. Aunque pueda parecer que realmente esto no es así y que muchos de los poderes tradicionales siguen manteniendo su estatus, si se hace el análisis desde una perspectiva histórica, se constata que realmente avanzamos hacia una sociedad más democrática y justa, donde las personas tenemos cada vez más capacidad de elegir. Se puede tomar el ejemplo de los matrimonios de conveniencia, una práctica bastante extendida en otras épocas que ahora incluso está mal vista por la mayor parte de la sociedad. Lo mismo sucede con el reparto de las tareas domésticas en el hogar, que, a pesar de estar muy lejos de una situación igualitaria, hace no mucho tiempo ni siquiera era susceptible de ser cuestionado. La función magistral del docente también se va viendo transformada, aumentando las fuentes de información y rompiendo con la exclusiva de un saber hermético, que a veces parecía inalcanzable.

La revolución de las TIC y la consecuente mejora de la educación a distancia, las clases semipresenciales $u$ otros procesos de aprendizaje en entornos virtuales implica una ruptura radical con el espacio y el tiempo tradicional del aula, un espacio que, en la mayoría de casos, ha sido concebido con un marcado carácter jerárquico y magistrocentrista, desde su propia construcción hasta la distribución del mobiliario. El profesorado ha ocupado normalmente el lugar central del aula, aquel hacia el que se orientan todas las mesas y pupitres, ordenadas en filas. El espacio el aula se ha constituido así como un auditorio donde el profesor o la profesora, los que saben, incluso desde una posición más elevada por una tarima, adoctrinan al alumnado, que no sabe. De esta manera, las interacciones entre el alumnado se han visto a veces dificultadas ya desde aspectos que pueden parecer meramente formales, como la estructura del aula. Las TIC establecen marcos para la interacción, como los mencionados fórums o los chats, a los que todo el mundo acude en pie de igualdad. El alumnado tiene la oportunidad de interactuar tanto con el profesorado como con sus 
compañeros y compañeras, sin barreras formales o psicológicas como tener que pedir la palabra o atreverse a hablar delante de un auditorio de cincuenta personas, o la falta de tiempo para poder contrastar todas las opiniones.

Diversificando las formas de participación, en un marco más horizontal y democrático, todas las personas pueden disponer de las mismas oportunidades para expresar sus ideas y aprender del diálogo con las demás. Es un contexto inmejorable para que el profesorado abandone su rol de experto o experta para convertirse en orientadores y dinamizadores de los debates en la red. En la línea de los cambios promovidos por la sociedad de la información, no se trata de que el profesorado transmita unos conocimientos que, mayoritariamente, están alcance de todos y todas, sino de que fomente la adquisición de las habilidades para seleccionar y procesar la información por parte de su alumnado.

Los recursos informáticos proporcionan una mayor transparencia en las informaciones dadas. El texto escrito, que es el soporte mayoritariamente utilizado en los entornos virtuales, permanece, de manera que cualquier persona puede acceder a él, en cualquier momento y desde cualquier lugar. Desde este punto de vista, los flujos de información entre profesorado, alumnado o cualquier otro miembro o estamento universitario serán mucho más transparentes si se efectúan en un entorno virtual, puesto que existe la posibilidad de que cualquiera que lo desee acceda a estas informaciones. También ayudan a desarrollar vías de innovación docente en relación a las modalidades de evaluación que se ofertan, permitiendo que el alumnado pueda construir su propio itinerario de acuerdo a intereses personales, necesidades o estilos de aprendizaje.

Finalmente, la universidad de hoy plantea un nuevo reto para el profesorado, que tiene la oportunidad de innovar la docencia de acuerdo a los cambios sociales, incentivar nuevos aprendizajes y crear sinergias con la motivación y creatividad del alumnado. La universidad también está transformando las posibilidades de acceso con la aplicación de las TIC, contribuyendo a la democratización de la institución. El desarrollo tecnológico de los últimos tiempos, junto con la unión de la voluntad empresarial, gubernamental y ciudadana para la generalización de las TIC, ha permitido su progresivo abaratamiento $y$, por consiguiente, la extensión de su uso a capas medias y bajas de la sociedad. Esto ha permitido acortar distancias y abrir una puerta para las personas que, por razones de carácter geográfico, laboral, familiar, personal o físico, no tenían la posibilidad de asistir a las clases presenciales regularmente. Ahora, estas personas pueden acceder, no sin muchos esfuerzos y renuncias, a unos estudios universitarios que, más adelante, podrán proporcionarles más y mejores oportunidades. Es por esta razón que hablamos de democratización de la universidad. Además, esta tendencia hacia la apertura y hacia la inclusión de más personas revierte en la propia sociedad en su conjunto, ya que el hecho de proporcionar más oportunidades de acceso a las titulaciones universitarias es, en el fondo, un modo de contribuir a la construcción de una sociedad más igualitaria y más justa.

\section{Conclusiones}

Que la universidad no camina al mismo ritmo de los cambios sociales es una realidad que podemos constatar en nuestras aulas todos los días. Por esta razón, hoy, más que nunca, se hace necesario un esfuerzo para que todos los avances que se introducen en otros ámbitos sociales, especialmente los promovidos por la utiliza- 
ción de las TIC, tengan una repercusión en la universidad. La innovación consiste en proporcionar las herramientas para que el alumnado pueda acceder a las bases de datos, a los programas de las mismas asignaturas en otras universidades, a las webs de los autores clave para ver su trabajo actual, para que pueda trabajar desde su propia casa en el espacio virtual, crear sus propias redes de intercambio de información e intereses intelectuales y académicos a través de la red. De esta manera, el alumnado participa activamente del proceso de aprendizaje y construcción del conocimiento conjuntamente con sus compañeras y compañeros y el profesorado. Este abanico de posibilidades que se abre entre los y las estudiantes les permiten adquirir más y nuevos aprendizajes a través de las interacciones y, en consecuencia, supone una importante contribución a la mejora de la calidad docente en la universidad.

\section{BIBLIOGRAFÍA}

AUBERT, A; DUQUE, E.; FISAS, M.; VALLS, R. (2004). Dialogar y transformar. Pedagogía crítica del siglo XXI. Barcelona: Graó.

Comisión de las Comunidades Europeas. 2001. Plan de acción e-Learning. Concebir la educación del futuro. Bruselas. Consulta de 3/9/03 en http://www.europa.eu.int/eur-lex/es/com/cnc/2001/com2001_0172es01.pdf

DELORS, J. 1996. La educación encierra un tesoro. Madrid: Santillana, Ediciones UNESCO.

ELBOJ, C.; PUIGDELLíVOL, I.; SOLER, M. y VALLS, R. (2002). Comunidades de aprendizaje. Transformar la educación. Barcelona: Graó.

FLECHA, R.; GÓMEZ, J. y PUIGVERT, L. (2001). Teoría sociológica contemporánea. Barcelona: Paidós.

GORZ, A. (1986). Los caminos del paraíso. Para comprender la crisis y salir de ella por la izquierda. Barcelona: Laia.

HABERMAS, J. (1987). Teoría de la acción comunicativa. Madrid: Taurus. 